\title{
Information-Theoretic Representation Learning for Positive-Unlabeled Classification
}

\author{
Tomoya Sakai ${ }^{* 1,2}$, Gang Niu* ${ }^{* 1,2}$, and Masashi Sugiyama*2,1 \\ ${ }^{1}$ Graduate School of Frontier Sciences, \\ The University of Tokyo, Japan \\ ${ }^{2}$ Center for Advanced Intelligence Project, \\ RIKEN, Japan
}

\begin{abstract}
Recent advances in weakly supervised classification allow us to train a classifier only from positive and unlabeled (PU) data. However, existing PU classification methods typically require an accurate estimate of the class-prior probability, which is a critical bottleneck particularly for high-dimensional data. This problem has been commonly addressed by applying principal component analysis in advance, but such unsupervised dimension reduction can collapse underlying class structure. In this paper, we propose a novel representation learning method from PU data based on the information-maximization principle. Our method does not require class-prior estimation and thus can be used as a preprocessing method for PU classification. Through experiments, we demonstrate that our method combined with deep neural networks highly improves the accuracy of PU class-prior estimation, leading to state-of-the-art PU classification performance.
\end{abstract}

\section{Introduction}

In real-world applications, it is conceivable that only positive and unlabeled (PU) data are available for training a classifier. For instance, in land-cover image

*The affiliation is as of March 2018. 
classification, images of urban regions can be easily labeled, while images of non-urban regions are difficult to annotate due to high diversity of non-urban regions containing, e.g., forest, seas, grasses, and soil (Li et al., 2011). To cope with such situations, PU classification has been actively studied (Letouzey et al., 2000; Elkan and Noto, 2008; du Plessis et al., 2015), and the state-of-the-art method allows us to systematically train deep neural networks only from PU data (Kiryo et al., 2017).

However, existing PU classification methods typically require an estimate of the class-prior probability, and their performance is sensitive to the quality of class-prior estimation (Kiryo et al., 2017). Although various class-prior estimation methods from PU data have been proposed so far (du Plessis and Sugiyama, 2014; Ramaswamy et al., 2016; Jain et al., 2016; du Plessis et al., 2017; Northcutt et al., 2017), accurate estimation of the class-prior is still highly challenging particularly for high-dimensional data.

In practice, principal component analysis is commonly used to reduce the data dimensionality in advance (Ramaswamy et al., 2016; du Plessis et al., 2017). However, such unsupervised dimension reduction completely abandons label information and thus the underlying class structure may be smashed. As a result, class-prior estimation often becomes even more difficult after dimension reduction.

The goal of this paper is to cope with this problem by proposing a representation learning method that can be executed only from PU data. Our method is developed within the framework of information maximization (Linsker, 1988).

Mutual information (MI) (Cover and Thomas, 2006) is a statistical dependency measure between random variables that is popularly used in informationtheoretic machine learning (Torkkola, 2003; Krause et al., 2010). However, empirically approximating MI from continuous-valued training data is not straightforward (Moon et al., 1995; Kraskov et al., 2004; Khan et al., 2007; Van Hulle, 2005; Suzuki et al., 2008) and is often sensitive to outliers (Basu et al., 1998; Sugiyama et al., 2012a). For this reason, we employ a squared-loss variant of mutual information (SMI) (Suzuki et al., 2009; Sugiyama, 2013), whose empirical estimator is known to be robust to outliers and possess superior numerical properties (Kanamori et al., 2012).

Our contributions are summarized as follows:

- We first develop a novel estimator of SMI that can be computed only from PU data, and prove its convergence to the optimal estimate of SMI in the optimal parametric rate when the linear-in-parameter model is used (Section 3).

- Based on this PU-SMI estimator, we then propose a representation learning method that can be executed without estimating the class-prior probabilities 
of unlabeled data (Section 4).

- Finally, we experimentally demonstrate that our PU representation learning method combined with deep neural networks highly improves the accuracy of PU class-prior estimation, and consequently the accuracy of PU classification can also be boosted significantly (Section 5).

\section{SMI}

In this section, we review the definition of ordinary MI and its variant, SMI.

Let $\boldsymbol{x} \in \mathbb{R}^{d}$ be an input pattern, $y \in\{ \pm 1\}$ be a corresponding class label, and $p(\boldsymbol{x}, y)$ be the underlying joint density, where $d$ is a positive integer.

Mutual information (MI) (Cover and Thomas, 2006) is a statistical dependency measure defined as

$$
\mathrm{MI}:=\sum_{y= \pm 1} \int p(\boldsymbol{x}, y) \log \left(\frac{p(\boldsymbol{x}, y)}{p(\boldsymbol{x}) p(y)}\right) \mathrm{d} \boldsymbol{x},
$$

where $p(\boldsymbol{x})$ is the marginal density of $\boldsymbol{x}$ and $p(y)$ is the probability mass of $y$. MI can be regarded as the Kullback-Leibler divergence from $p(\boldsymbol{x}, y)$ to $p(\boldsymbol{x}) p(y)$, and therefore MI is non-negative and takes zero if and only if $p(\boldsymbol{x}, y)=p(\boldsymbol{x}) p(y)$, i.e., $\boldsymbol{x}$ and $y$ are statistically independent. This property allows us to evaluate the dependency between $\boldsymbol{x}$ and $y$. However, empirically approximating MI from continuous data is not straightforward (Moon et al., 1995; Kraskov et al., 2004; Khan et al., 2007; Van Hulle, 2005; Suzuki et al., 2008) and is often sensitive to outliers (Basu et al., 1998; Sugiyama et al., 2012a).

To cope with this problem, squared-loss MI (SMI) has been proposed (Suzuki et al., 2009), which is a squared-loss variant of MI defined as

$$
\mathrm{SMI}:=\sum_{y= \pm 1} \frac{p(y)}{2} \int\left(\frac{p(\boldsymbol{x}, y)}{p(\boldsymbol{x}) p(y)}-1\right)^{2} p(\boldsymbol{x}) \mathrm{d} \boldsymbol{x} .
$$

SMI can be regarded as the Pearson divergence (Pearson, 1990) from $p(\boldsymbol{x}, y)$ to $p(\boldsymbol{x}) p(y)$. SMI is also non-negative and takes zero if and only if $\boldsymbol{x}$ and $y$ are independent.

So far, methods for estimating SMI from positive and negative samples and SMI-based machine learning algorithms have been explored extensively, and their effectiveness has been demonstrated (Sugiyama, 2013). 


\section{SMI Estimation from PU Data}

The goal of this paper is to develop a representation learning method from PU data. To this end, we propose an estimator of SMI that can be computed only from PU data in this section.

\subsection{SMI with PU Data}

Suppose that we are given PU data (Ward et al., 2009):

$$
\begin{aligned}
& \left\{\boldsymbol{x}_{i}^{\mathrm{P}}\right\}_{i=1}^{n_{\mathrm{P}}} \stackrel{\text { i.i.d. }}{\sim} p(\boldsymbol{x} \mid y=+1), \\
& \left\{\boldsymbol{x}_{k}^{\mathrm{U}}\right\}_{k=1}^{n_{\mathrm{U}}} \stackrel{\text { i.i.d. }}{\sim} p(\boldsymbol{x})=\theta_{\mathrm{P}} p(\boldsymbol{x} \mid y=+1)+\theta_{\mathrm{N}} p(\boldsymbol{x} \mid y=-1),
\end{aligned}
$$

where $\theta_{\mathrm{P}}:=p(y=+1)$ and $\theta_{\mathrm{N}}:=p(y=-1)$ are the class-prior probabilities.

First, we express SMI in Eq. (1) in terms of only the densities of PU data, without negative data (see Appendix A for its proof):

Theorem 1. Let

$$
\text { PU-SMI }:=\frac{\theta_{\mathrm{P}}}{2 \theta_{\mathrm{N}}} \int\left(\frac{p(\boldsymbol{x} \mid y=+1)}{p(\boldsymbol{x})}-1\right)^{2} p(\boldsymbol{x}) \mathrm{d} \boldsymbol{x} .
$$

Then we have $\mathrm{PU}-\mathrm{SMI}=\mathrm{SMI}$.

If PU densities $p(\boldsymbol{x} \mid y=+1)$ and $p(\boldsymbol{x})$ are estimated from PU data, the above PU-SMI allows us to approximate SMI only from PU data. However, such a naive approach works poorly due to hardness of density estimation and computing the ratio of estimated densities further magnifies the estimation error (Sugiyama et al., 2012b).

\subsection{PU-SMI Estimation}

Here, we propose a more sophisticated approach to estimating PU-SMI from PU data.

First, we give the following theorem, which gives a lower-bound of PU-SMI (see Appendix B for its proof):

Theorem 2. For any function $f(\boldsymbol{x})$,

$$
\text { PU-SMI } \geq \frac{\theta_{\mathrm{P}}}{\theta_{\mathrm{N}}}\left(-J_{\mathrm{PU}}(f)-\frac{1}{2}\right),
$$


where

$$
J_{\mathrm{PU}}(f):=\frac{1}{2} \int f^{2}(\boldsymbol{x}) p(\boldsymbol{x}) \mathrm{d} \boldsymbol{x}-\int f(\boldsymbol{x}) p(\boldsymbol{x} \mid y=+1) \mathrm{d} \boldsymbol{x},
$$

and the equality holds if and only if

$$
f(\boldsymbol{x})=\frac{p(\boldsymbol{x} \mid y=+1)}{p(\boldsymbol{x})} .
$$

While PU-SMI itself contains $p(\boldsymbol{x} \mid y=+1)$ and $p(\boldsymbol{x})$ in a complicated way, the lower bound consists only of the expectations over $p(\boldsymbol{x} \mid y=+1)$ and $p(\boldsymbol{x})$. Thus, the lower bound can be immediately approximated empirically.

Based on this theorem, we maximize an empirical approximation to the lower bound (3), which is expressed as

$$
\widehat{w}:=\underset{w \in \mathcal{W}}{\operatorname{argmin}} \widehat{J}_{\mathrm{PU}}(w),
$$

where

$$
\widehat{J}_{\mathrm{PU}}(w):=\frac{1}{2 n_{\mathrm{U}}} \sum_{k=1}^{n_{\mathrm{U}}} w^{2}\left(\boldsymbol{x}_{k}^{\mathrm{U}}\right)-\frac{1}{n_{\mathrm{P}}} \sum_{i=1}^{n_{\mathrm{P}}} w\left(\boldsymbol{x}_{i}^{\mathrm{P}}\right)
$$

and $\mathcal{W}$ is a (user-defined) function class such as linear-in-parameter models, kernel models, and neural networks. In this optimization, we can drop the unknown class-prior ratio $\theta_{\mathrm{P}} / \theta_{\mathrm{N}}$, which is difficult to estimate accurately (du Plessis and Sugiyama, 2014; Ramaswamy et al., 2016; Jain et al., 2016; du Plessis et al., 2017).

Finally, our PU-SMI estimator is given as

$$
\widehat{\mathrm{PU}-\mathrm{SMI}}=\frac{\theta_{\mathrm{P}}}{\theta_{\mathrm{N}}}\left(-\widehat{\mathrm{J}_{\mathrm{PU}}}(\widehat{w})-\frac{1}{2}\right) .
$$

$\widehat{\mathrm{PU}-\mathrm{SM}} \mathrm{includes}$ the class-prior ratio $\theta_{\mathrm{P}} / \theta_{\mathrm{N}}$ only as a proportional constant. Therefore, class-prior estimation is not needed when we just want to maximize or minimize PU-SMI. We will utilize this excellent property in Section 4 when we develop a representation learning method.

Note that if $W$ contains the true density-ratio function $p(\boldsymbol{x} \mid y=+1) / p(\boldsymbol{x})$, $\widehat{\mathrm{PU}-\mathrm{SMI}} \rightarrow$ PU-SMI $\left(n_{\mathrm{P}}, n_{\mathrm{U}} \rightarrow \infty\right)$ with some regularity condition. On the other hand, if the function class does not contain the true density-ratio function, there is a gap between PU-SMI and PU-SMI even if $n_{\mathrm{P}}, n_{\mathrm{U}} \rightarrow \infty$. Such a gap often arises in real-world applications because a function class does not always include the true density-ratio function. However, the gap may not be a critical issue in practice as long as a reasonably flexible function class is chosen, as demonstrated by the experiments in Section 5. Even though the gap may exist in practical implementation, we show that the classification performance can be improved by our proposed representation learning method. 


\subsection{Analytic Solution for Linear-in-Parameter Models}

Our SMI estimator is applicable to any density-ratio model $w$.

If a neural network is used as $w$, the solution may be obtained by a stochastic gradient method (Goodfellow et al., 2016; Abadi et al., 2015; Jia et al., 2014).

Another candidate of the density-ratio model is a linear-in-parameter model:

$$
w(\boldsymbol{x})=\sum_{\ell=1}^{b} \beta_{\ell} \phi_{\ell}(\boldsymbol{x})=\boldsymbol{\beta}^{\top} \phi(\boldsymbol{x}),
$$

where $\boldsymbol{\beta}:=\left(\beta_{1}, \ldots, \beta_{b}\right)^{\top} \in \mathbb{R}^{b}$ is a vector of parameters, ${ }^{\top}$ denotes the transpose, $b$ is the number of parameters, and $\boldsymbol{\phi}(\boldsymbol{x}):=\left(\phi_{1}(\boldsymbol{x}), \ldots, \phi_{b}(\boldsymbol{x})\right)^{\top} \in \mathbb{R}^{b}$ is a vector of basis functions. This model allows us to obtain an analytic-form PUSMI estimator. Furthermore, the optimal convergence is theoretically guaranteed as shown in Section 3.4.

When the $\ell_{2}$-regularizer is included, the optimization problem yields

$$
\widehat{\boldsymbol{\beta}}:=\underset{\boldsymbol{\beta}}{\operatorname{argmin}} \frac{1}{2} \boldsymbol{\beta}^{\top} \widehat{\boldsymbol{H}}^{\mathrm{U}} \boldsymbol{\beta}-\boldsymbol{\beta}^{\top} \widehat{\boldsymbol{h}}^{\mathrm{P}}+\frac{\lambda_{\mathrm{PU}}}{2}\|\boldsymbol{\beta}\|_{2}^{2},
$$

where $\lambda_{\mathrm{PU}} \geq 0$ is the regularization parameter, $\|\cdot\|_{2}$ denotes the $\ell_{2}$-norm, and

$$
\begin{aligned}
\widehat{H}_{\ell, \ell^{\prime}}^{\mathrm{U}} & :=\frac{1}{n_{\mathrm{U}}} \sum_{k=1}^{n_{\mathrm{U}}} \phi_{\ell}\left(\boldsymbol{x}_{k}^{\mathrm{U}}\right) \phi_{\ell^{\prime}}\left(\boldsymbol{x}_{k}^{\mathrm{U}}\right), \\
\widehat{h}_{\ell}^{\mathrm{P}} & :=\frac{1}{n_{\mathrm{P}}} \sum_{i=1}^{n_{\mathrm{P}}} \phi_{\ell}\left(\boldsymbol{x}_{i}^{\mathrm{P}}\right) .
\end{aligned}
$$

Note that $\widehat{H}_{\ell, \ell^{\prime}}^{\mathrm{U}}$ is the $\left(\ell, \ell^{\prime}\right)$-th element of $\widehat{\boldsymbol{H}}^{\mathrm{U}}$ and $\widehat{h}_{\ell}^{\mathrm{P}}$ is the $\ell$-th element of $\widehat{\boldsymbol{h}}^{\mathrm{P}}$. The solution can be obtained analytically by differentiating the objective function with respect to $\boldsymbol{\beta}$ and set it to zero: $\widehat{\boldsymbol{\beta}}=\left(\widehat{\boldsymbol{H}}^{\mathrm{U}}+\lambda_{\mathrm{PU}} \boldsymbol{I}_{b}\right)^{-1} \widehat{\boldsymbol{h}}^{\mathrm{P}}$. Finally, with the obtained estimator, we can compute an SMI approximator only from positive and unlabeled data:

$$
\widehat{\mathrm{PU}-\mathrm{SMI}}=\frac{\theta_{\mathrm{P}}}{\theta_{\mathrm{N}}}\left(\widehat{\boldsymbol{\beta}}^{\top} \widehat{\boldsymbol{h}}^{\mathrm{P}}-\frac{1}{2} \widehat{\boldsymbol{\beta}}^{\top} \widehat{\boldsymbol{H}}^{\mathrm{U}} \widehat{\boldsymbol{\beta}}-\frac{1}{2}\right) .
$$

Note that all hyper-parameters such as the regularization parameter can be tuned by the value of $J_{\mathrm{PU}}$ approximated by (cross-)validation samples.

\subsection{Convergence Analysis}

Here we analyze the convergence rate of learned parameters of the density-ratio model and the PU-SMI approximator based on the perturbation analysis of 
optimization problems (Bonnans and Cominetti, 1996; Bonnans and Shapiro, 1998).

In our theoretical analysis, we focus on the linear-in-parameter model in Eq. (6). We first define $\boldsymbol{\beta}^{* \top} \boldsymbol{\phi}(\boldsymbol{x})$ as the minimizer of the expected error, i.e., $\boldsymbol{\beta}^{*}:=\operatorname{argmin}_{\boldsymbol{\beta} \in \mathbb{R}^{b}} J_{\mathrm{PU}}(\boldsymbol{\beta})$ and denote its estimator by $\widehat{\boldsymbol{\beta}}=$ $\operatorname{argmin}_{\boldsymbol{\beta} \in \mathbb{R}^{b}} \widehat{J}_{\mathrm{PU}}(\boldsymbol{\beta})$ in this analysis. Note that the linear-in-parameter model is assumed as a simple baseline for theoretical analysis.

For the linear-in-parameter model, we assume that the basis functions satisfy $0 \leq \phi_{\ell}(\boldsymbol{x}) \leq 1$ for all $\ell=1, \ldots, b$, and $\widehat{\boldsymbol{H}}^{\mathrm{U}}$ and $\boldsymbol{H}^{\mathrm{U}}$ are positive definite matrices.

Let

$$
\text { PU-SMI* }:=\frac{\theta_{\mathrm{P}}}{\theta_{\mathrm{N}}}\left(-J_{\mathrm{PU}}\left(\boldsymbol{\beta}^{*}\right)-\frac{1}{2}\right)
$$

be the PU-SMI with $\boldsymbol{\beta}^{*}$. Similarly, P $\widehat{\mathrm{U}-\mathrm{SM}} \mathrm{I}$ is the estimate of the PU-SMI with $\widehat{\boldsymbol{\beta}}$. Let $\mathcal{O}_{p}$ denote the order in probability. Then we have the following convergence results (its proof is given in Appendix C):

Theorem 3. As $n_{\mathrm{P}}, n_{\mathrm{U}} \rightarrow \infty$, we have

$$
\begin{aligned}
\left\|\widehat{\boldsymbol{\beta}}-\boldsymbol{\beta}^{*}\right\|_{2} & =\mathcal{O}_{p}\left(1 / \sqrt{n_{\mathrm{P}}}+1 / \sqrt{n_{\mathrm{U}}}\right), \\
\mid \mathrm{PU}-\mathrm{SMI}^{*}-\mathrm{PU}-\mathrm{SMI} & =\mathcal{O}_{p}\left(1 / \sqrt{n_{\mathrm{P}}}+1 / \sqrt{n_{\mathrm{U}}}\right) .
\end{aligned}
$$

Theorem 3 guarantees that the convergence of the density-ratio estimator and the PU-SMI approximator. In our setting, since $n_{\mathrm{P}}$ and $n_{\mathrm{U}}$ can increase independently, this is the optimal convergence rate without any additional assumption (Kanamori et al., 2009, 2012).

Theorem 3 shows that both positive and unlabeled data contribute to convergence. This implies that unlabeled data is directly used in the estimation rather than extracting the information of a data structure, such as the cluster structure frequently assumed in semi-supervised learning (Chapelle et al., 2006). The theorem also shows that the convergence rate of our method is dominated by the smaller size of positive or unlabeled data.

Note that since this analysis focuses on the linear-in-parameter model, there might be a gap between PU-SMI and PU-SMI*, implying that PU-SMI < PU-SMI*. The convergence analysis guarantees that PU-SMI with the linear-inparameter model converges to $\mathrm{PU}_{-} \mathrm{SMI}^{*}$, but there might be an approximation error (Mohri et al., 2012), as discussed in Section 3.2. 


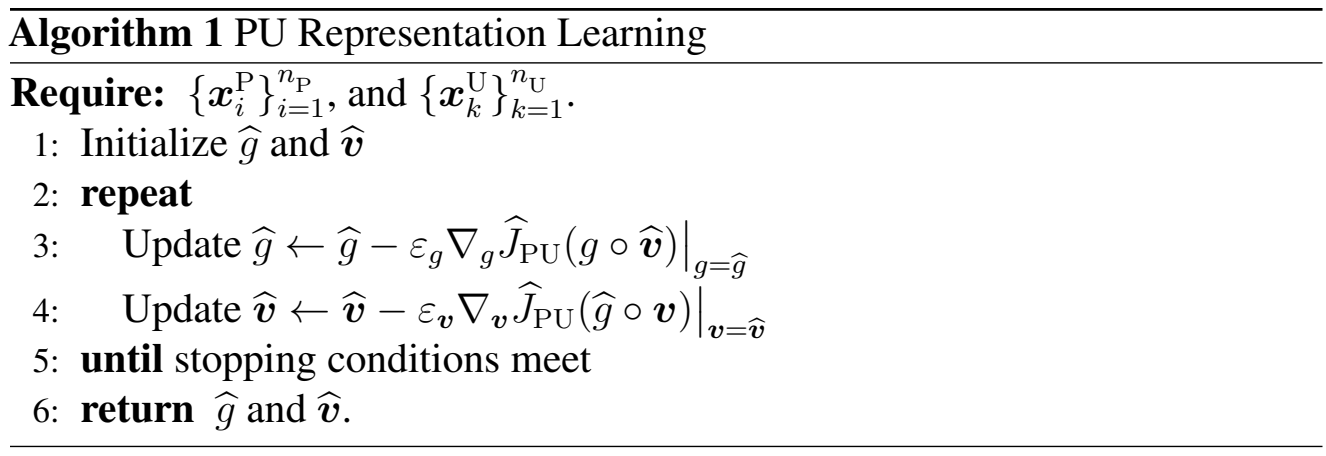

\section{PU Representation Learning}

In this section, we propose a representation learning method based on PU-SMI maximization. We extend the existing SMI-based dimension reduction (Suzuki and Sugiyama, 2013), called least-squares dimension reduction (LSDR), to PU representation learning. While LSDR only considers linear dimension reduction, we extend it to non-linear dimension reduction by neural networks.

Let $\boldsymbol{v}: \mathbb{R}^{d} \rightarrow \mathbb{R}^{m}$, where $m<d$, be a mapping from an input vector to its low-dimensional representation. If the mapping function satisfies

$$
p(y \mid \boldsymbol{x})=p(y \mid \boldsymbol{v}(\boldsymbol{x})),
$$

the obtained low-dimensional representation can be used as the new input instead of the original input vector. Finding the mapping function satisfying the condition (7) is known as sufficient dimension reduction (Li, 1991). Let SMI be

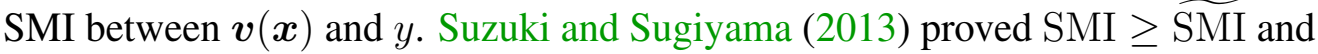
equality holds when the condition (7) is satisfied. That is, maximizing SMI is finding sufficient representation for the output $y$.

Following the information-maximization principle (Linsker, 1988), we maximize PU-SMI with respect to the mapping to find low-dimensional representation that maximally preserves dependency between input and output.

More specifically, since P $\widehat{\mathrm{U}-\mathrm{SM}}=-\theta_{\mathrm{P}} / \theta_{\mathrm{N}} \cdot \min _{w \in \mathcal{W}} \widehat{\widehat{J}_{\mathrm{PU}}}(w)-\theta_{\mathrm{P}} /\left(2 \theta_{\mathrm{N}}\right)$, we minimize $\widehat{J}_{\mathrm{PU}}(w)$ with respect to $w$. Furthermore, inspired by the alternative optimization algorithm for the SMI-based dimension reduction method (Suzuki and Sugiyama, 2013), we decompose $w$ into $g$ and $\boldsymbol{v}$ such that $w=g \circ \boldsymbol{v}$ and minimize $\widehat{J}_{\mathrm{PU}}(g \circ \boldsymbol{v})$ by optimizing $g$ and $\boldsymbol{v}$ alternatively, where $g: \mathbb{R}^{m} \rightarrow \mathbb{R}$ and "०" denotes the function composition, i.e., $(g \circ \boldsymbol{v})(\boldsymbol{x})=g(\boldsymbol{v}(\boldsymbol{x}))$. In this decomposition, $\boldsymbol{v}$ can be regarded as a mapping function extracting features from input pattern and $g$ a density ratio function $p(\boldsymbol{x} \mid y=+1) / p(\boldsymbol{x})$. First, we approximate SMI by minimizing Eq. (4) with respect to density ratio $g$ with 
current mapping $\widehat{\boldsymbol{v}}$ fixed:

$$
\widehat{g}=\underset{g}{\operatorname{argmin}} \widehat{J}_{\mathrm{PU}}(g \circ \widehat{\boldsymbol{v}}) .
$$

Then, we update mapping $\widehat{\boldsymbol{v}}$ to increase the estimated PU-SMI with current density ratio $\widehat{g}$ fixed:

$$
\widehat{\boldsymbol{v}} \leftarrow \widehat{\boldsymbol{v}}-\varepsilon \nabla_{\boldsymbol{v}} \widehat{J}_{\mathrm{PU}}(\widehat{g} \circ \boldsymbol{v}),
$$

where $\varepsilon$ is the step size. This process is repeated until convergence. In practice, we may alternately optimize $g$ and $\boldsymbol{v}$ as described in Algorithm 1 to simplify the implementation. ${ }^{*}$ We refer to our representation learning method for PU data as positive-unlabeled representation learning (PURL).

Note again that, in the above optimization process, unknown class-prior ratio $\theta_{\mathrm{P}} / \theta_{\mathrm{N}}$ does not need to be estimated in advance, which is a significant advantage of the proposed method.

\section{Experiments}

In this section, we experimentally investigate the behavior of the proposed PUSMI estimator and evaluate the performance of the proposed representation learning method on various benchmark datasets.

\subsection{Accuracy of PU-SMI Estimation}

First, we investigate the estimation accuracy of the proposed PU-SMI estimator on datasets obtained from the LIBSVM webpage (Chang and Lin, 2011).

As the model $w$, we use the linear-in-parameter model with the Gaussian basis functions $\phi_{\ell}(\boldsymbol{x}):=\exp \left(-\left\|\boldsymbol{x}-\boldsymbol{x}_{\ell}\right\|^{2} /\left(2 \sigma^{2}\right)\right)$ for $\ell=1, \ldots, b$, where $\sigma>0$ is the bandwidth and $\left\{\boldsymbol{x}_{\ell}\right\}_{\ell=1}^{b}$ are the centers of the Gaussian functions randomly sampled from $\left\{\boldsymbol{x}_{k}^{\mathrm{U}}\right\}_{k=1}^{n_{\mathrm{U}}}$. The Gaussian bandwidth and the $\ell_{2}$-regularization parameter are determined by five-fold cross-validation. We vary the number of positive/unlabeled samples from 10 to 200, with the number of unlabeled/positive samples fixed. The class-prior was assumed to be known in this illustrative experiment and set at $\theta_{\mathrm{P}}=0.5$.

Figure 1 summarizes the average and standard error of the squared estimation error of PU-SMI over 50 trials. ${ }^{\dagger}$ This shows that the mean squared error decreases

\footnotetext{
${ }^{*}$ We also tried to optimize $w$ and $\boldsymbol{v}$ simultaneously. That is, $J_{\mathrm{PU}}$ is minimized with respect to $g$ without decomposing $g$ into $w$ and $\boldsymbol{v}$, but it did not work well in our preliminary experiments.

${ }^{\dagger}$ We compute the squared error between the estimated PU-SMI and the supervised SMI estimator (Suzuki et al., 2009) with a sufficiently large number of positive and negative samples.
} 


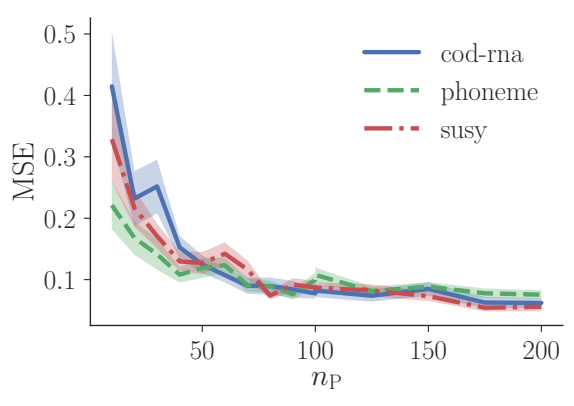

(a) $n_{\mathrm{U}}=400$

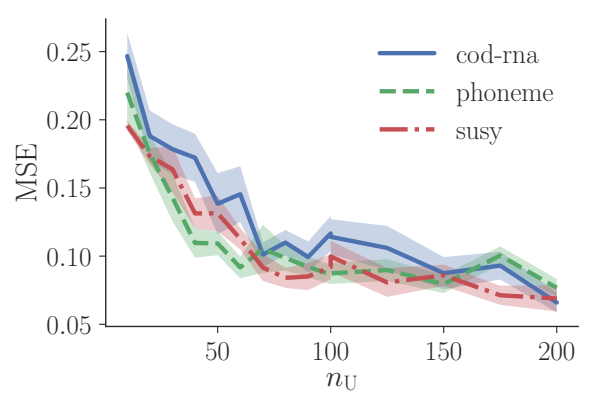

(b) $n_{\mathrm{P}}=200$

Figure 1: Average and standard error of the squared estimation error of PU-SMI over 50 trials. (a) $n_{\mathrm{P}}$ is increased while $n_{\mathrm{U}}=400$ is fixed. (b) $n_{\mathrm{U}}$ is increased while $n_{\mathrm{P}}=200$ is fixed. The results show that both positive and unlabeled samples contribute to improving the estimation accuracy of SMI.

both when the number of positive samples is increased and the number of unlabeled samples is increased. Therefore, both positive and unlabeled data contribute to improving the estimation accuracy of SMI, which well agrees with our theoretical analysis in Section 3.4.

\subsection{Representation Learning}

Next, we evaluate the performance of the proposed representation learning method, PURL.

Illustration: We first illustrate how our proposed method works on an artificial data set. We generate samples from the following densities:

$$
\begin{aligned}
& p(\boldsymbol{x} \mid y=+1)=N\left(\boldsymbol{x} ;\left(\begin{array}{l}
0 \\
0
\end{array}\right),\left(\begin{array}{cc}
0.25 & 0 \\
0 & 4
\end{array}\right)\right), \\
& p(\boldsymbol{x} \mid y=-1)=\frac{1}{2} N\left(\boldsymbol{x} ;\left(\begin{array}{l}
3 \\
0
\end{array}\right),\left(\begin{array}{cc}
0.25 & 0 \\
0 & 4
\end{array}\right)\right)+\frac{1}{2} N\left(\boldsymbol{x} ;\left(\begin{array}{c}
-3 \\
0
\end{array}\right),\left(\begin{array}{cc}
0.25 & 0 \\
0 & 4
\end{array}\right)\right),
\end{aligned}
$$

where $N(\boldsymbol{x} ; \boldsymbol{\mu}, \boldsymbol{\Sigma})$ is the normal density with the mean vector $\boldsymbol{\mu}$ and the covariance matrix $\Sigma$. The class-prior is set at $\theta_{\mathrm{P}}=0.7$. From the densities, we draw $n_{\mathrm{P}}=400$ positive and $n_{\mathrm{U}}=1000$ unlabeled samples. For comparison, we apply PCA, Fisher’s discriminant analysis (FDA), and PNRL (the supervised counterpart of PURL) to the data. As the label information for FDA and PNRL, $\mathrm{U}$ data is simply regarded as $\mathrm{N}$ data even though $\mathrm{U}$ data is a mixture of $\mathrm{P}$ and

\footnotetext{
The details of PNRL are described in Appendix E.
} 


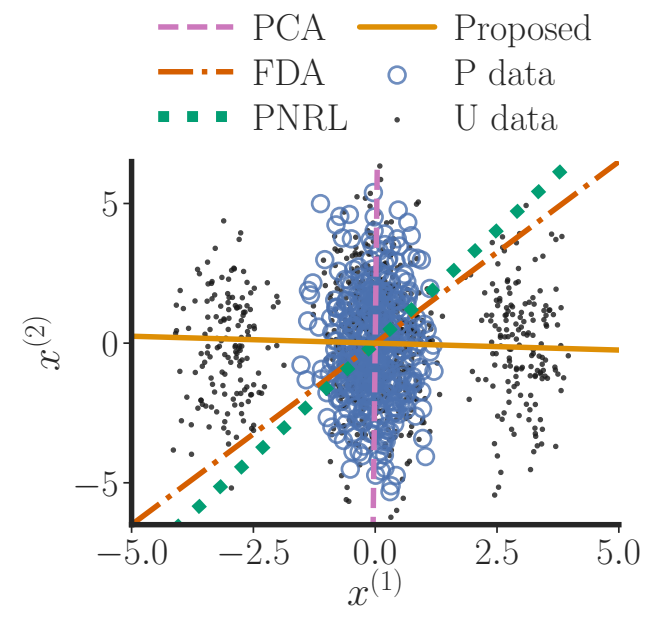

(a) Data and obtained subspaces

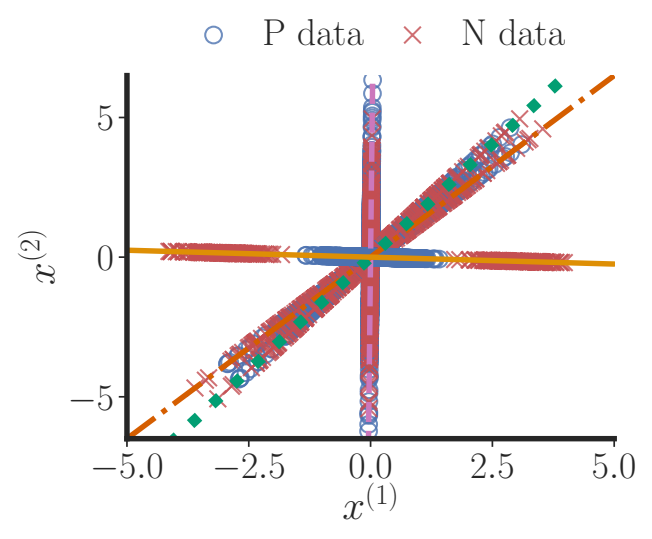

(b) Projected data with its labels

Figure 2: (a) Positive and unlabeled data. The estimated subspaces obtained by PCA, FDA, PNRL, and our proposed method. (b) Unlabeled data with the true labels projected onto the subspaces obtained by PCA, FDA, and our method, respectively. The results indicate that PCA, FDA, and PNRL smash underlying class structure, while the positive and negative labels are visibly separated in the subspace obtained by our method.

$\mathrm{N}$ data. Since PCA and FDA are linear transformations, we also use a linear transformation in PNRL and PURL for this numerical illustration. Specifically, we use a two-layer perceptron for $w$. The first fully-connected layer is used as linear transformation to obtain one-dimensional representation. The rectified linear unit (ReLU) (Glorot et al., 2011) is used for activation functions of the output of the first layer, which can be seen as feature mapping functions in the linear-in-parameter model. The second layer is just a single connection that weighs the output of the first layer.

We plot the subspaces obtained by PCA, FDA, PNRL, and our proposed method in Figure 2(a). Since the data is distributed vertically, the subspace obtained by PCA is almost parallel to the vertical axis (the dashed line). FDA and PNRL return diagonal lines (the dashdot and dotted lines), showing that regarding $\mathrm{U}$ data as $\mathrm{N}$ data is not an appropriate way. On the other hand, the subspace obtained by our method is almost parallel to the horizontal axis (the solid line). Figure 2(b) plots projected labeled data onto those subspaces. This shows that the labels of the data projected by PCA, FDA, and PNRL are hardly distinguishable due to significant overlap, which makes class-prior estimation very hard. In contrast, we can easily separate the classes of samples projected by the proposed method, which eases class-prior estimation. 
Table 1: Average absolute error (with standard error) between the estimated class-prior and the true value on benchmark datasets over 20 trials. None means that the class-prior is estimated without dimension reduction methods, PCA is the principal component analysis, FDA is Fisher's discriminant analysis, and PNRL is the supervised counterpart of the proposed method. The class-prior is estimated by the method based on kernel mean embedding. The boldface denotes the best and comparable approaches in terms of the average absolute error according to the t-test at the significance level $5 \%$.

\begin{tabular}{|c|c|c|c|c|c|c|c|c|}
\hline \multirow{2}{*}{ Dataset } & \multirow{2}{*}{$\theta_{\mathrm{P}}$} & \multirow{2}{*}{ None } & \multicolumn{3}{|c|}{ PCA } & \multirow{2}{*}{ FDA } & \multirow{2}{*}{ PNRL } & \multirow{2}{*}{ PURL } \\
\hline & & & $\lfloor d / 4\rfloor$ & $\lfloor d / 2\rfloor$ & $\lfloor 3 d / 4\rfloor$ & & & \\
\hline \multirow{3}{*}{ ijcnn1 } & 0.3 & ) & $0.26(0.11)$ & $(0.11)$ & 0 & 0.03 & $0.20(0$. & $0.21(0.07)$ \\
\hline & 0.5 & $0.18(0.05)$ & $0.14(0.09)$ & $0.14(0.09)$ & $0.17(0.06)$ & $0.04(0.01)$ & $0.21(0.08)$ & $0.19(0.07)$ \\
\hline & 0.7 & $0.08(0.01)$ & $0.11(0.05)$ & $0.11(0.05)$ & $0.10(0.04)$ & $0.07(0.01)$ & $0.11(0.05)$ & $0.10(0.01)$ \\
\hline \multirow{3}{*}{ phishing } & 0 & $02(0.00)$ & $02(0,00)$ & $0.02(0.00)$ & 0.02 & 0.04 & 003 & $.00)$ \\
\hline & 0.5 & 0 & 01 & 0.01 & 0.01 & $0.07(0$. & $0.04(0$ & $0.03(0.02)$ \\
\hline & 0.7 & $0.02(0.00)$ & $0.02(0.00)$ & $0.02(0.00)$ & $0.02(0.00)$ & $0.11(0.04)$ & $0.05(0.03)$ & $0.02(0.00)$ \\
\hline \multirow{3}{*}{ mushrooms } & 0.3 & $05(001)$ & $05(0.01)$ & $0.05(0.01)$ & 0, & $0.09(0.03)$ & $0.03(0.00)$ & $0.03(0.00)$ \\
\hline & 0.5 & 0.0 & ) & $0.05(0.01)$ & 0.05 & 0 . & 1) & 0.04 \\
\hline & 0.7 & $0.03(0.01)$ & $0.03(0.00)$ & $0.03(0.00)$ & $0.03(0.01)$ & $0.20(0.06)$ & $0.03(0.00)$ & $0.04(0.03)$ \\
\hline \multirow{3}{*}{ a9a } & 0.3 & $011(0 \Omega 2)$ & $11(02)$ & $0.11(0.02)$ & (1) & $0.05(0.01)$ & $0.08(0.03)$ & $0.00)$ \\
\hline & 0.5 & $0.10(0.02)$ & $0.10(0.02)$ & $0.10(0.02)$ & $0.10(0.02)$ & $0.09(0.04)$ & $0.09(0.03)$ & $0.04(0.01)$ \\
\hline & 0.7 & $0.08(0.03)$ & $0.08(0.03)$ & $0.08(0.03)$ & $0.08(0.03)$ & $0.18(0.06)$ & $0.08(0.03)$ & $0.04(0.01)$ \\
\hline \multirow{3}{*}{ MNIST } & 0.3 & ) & (200) & (0) & 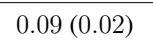 & $0.27(0.01)$ & $0.01(0.00)$ & $0.05(0.02)$ \\
\hline & 0.5 & $0.15(0.11)$ & $0.15(0.11)$ & $0.15(0.11)$ & $0.15(0.11)$ & $0.46(0.01)$ & $0.03(0.00)$ & $0.06(0.03)$ \\
\hline & 0.7 & $0.60(0.21)$ & $0.60(0.21)$ & $0.60(0.21)$ & $0.60(0.21)$ & $0.65(0.02)$ & $0.06(0.01)$ & $0.07(0.01)$ \\
\hline \multirow{3}{*}{ F-MNIST } & 0.3 & $0.02(0.00)$ & $0.02(0.00)$ & $0.02(0.00)$ & $0.02(0.00)$ & $0.25(0.01)$ & $0.03(0.00)$ & $0.03(0.00)$ \\
\hline & 0.5 & $0.03(0.00)$ & $0.03(0.00)$ & $0.03(0.00)$ & $0.03(0.00)$ & $0.45(0.01)$ & $0.02(0.00)$ & $0.04(0.03)$ \\
\hline & 0.7 & $0.03(0.00)$ & $0.03(0.00)$ & $0.03(0.00)$ & $0.03(0.00)$ & $0.66(0.02)$ & $0.02(0.00)$ & $0.07(0.03)$ \\
\hline \multirow{3}{*}{20 News } & 0.3 & 0. & ) & $0.04(0.00)$ & $0.04(0.00)$ & $0.28(0.00)$ & $0.29(0.09)$ & $0.03(0.01)$ \\
\hline & 0.5 & $0.08(0.03)$ & $0.06(0.01)$ & $0.07(0.01)$ & $0.08(0.03)$ & $0.49(0.00)$ & $0.25(0.07)$ & $0.05(0.01)$ \\
\hline & 0.7 & $0.69(0.00)$ & $0.69(0.00)$ & $0.69(0.00)$ & $0.69(0.00)$ & $0.69(0.00)$ & $0.13(0.03)$ & $0.07(0.01)$ \\
\hline
\end{tabular}


Table 2: Average misclassification rates (with standard error) on benchmark datasets over 20 trials. The boldface denotes the best and comparable approaches in terms of the average absolute error according to the t-test at the significance level $5 \%$.

\begin{tabular}{|c|c|c|c|c|c|c|c|c|}
\hline \multirow{2}{*}{ Dataset } & \multirow{2}{*}{$\theta_{\mathrm{P}}$} & \multirow{2}{*}{ None } & \multicolumn{3}{|c|}{ PCA } & \multirow{2}{*}{ FDA } & \multirow{2}{*}{ PNRL } & \multirow{2}{*}{ PURL } \\
\hline & & & $\lfloor d / 4\rfloor$ & $\lfloor d / 2\rfloor$ & $\lfloor 3 d / 4\rfloor$ & & & \\
\hline \multirow{3}{*}{ ijcnn1 } & 0.3 & $25.32(1.23)$ & $27.79(2.05)$ & $27.79(2.05)$ & $29.21(1.30)$ & $7.00(0.59)$ & $28.57(2.52)$ & $25.92(2.64)$ \\
\hline & 0.5 & $21.43(1.22)$ & $17.88(1.72)$ & $17.88(1.72)$ & $20.75(1.15)$ & $8.25(1.33)$ & $26.52(2.26)$ & $21.52(1.69)$ \\
\hline & 0.7 & $12.07(0.53)$ & $14.94(1.02)$ & $14.94(1.02)$ & 14.61 & $11.23(1.34)$ & $17.34(1.58)$ & $13.70(1.04)$ \\
\hline \multirow{3}{*}{ phishing } & 0.3 & $7.41(0.46)$ & $7.46(0.48)$ & $7.46(0.48)$ & $7.57(0.46)$ & $10.30(2.42)$ & $11.09(0.98)$ & $7.62(0.45)$ \\
\hline & 0.5 & $12.85(2.11)$ & $9.75(0.46)$ & $9.75(0.46)$ & $9.82(0.40)$ & $24.43(3.09)$ & $32.02(3.05)$ & $10.05(0.46)$ \\
\hline & 0.7 & $8.07(0.44)$ & $8.85(1.08)$ & $8.85(1.08)$ & $7.63(0.37)$ & $25.62(1.40)$ & $29.04(0.73)$ & $8.02(0.37)$ \\
\hline \multirow{3}{*}{ mushrooms } & 0.3 & $0.73(0.20)$ & $1.15(0.58)$ & $0.57(0.14)$ & $0.49(0.14)$ & $1.52(0.36)$ & $0.24(0.06)$ & $0.43(0.09)$ \\
\hline & 0.5 & $0.57(0.11)$ & $0.57(0.11)$ & $0.78(0.16)$ & $0.57(0.11)$ & $3.40(0.47)$ & $1.10(0.24)$ & $3.40(2.39)$ \\
\hline & 0.7 & $1.42(0.28)$ & $1.42(0.28)$ & $1.50(0.27)$ & $1.42(0.28)$ & $6.38(0.66)$ & $1.40(0.27)$ & $1.61(0.48)$ \\
\hline \multirow{3}{*}{ a9a } & 0.3 & $24.93(1.19)$ & $26.49(1.89)$ & $26.49(1.89)$ & $26.20(1.73)$ & $21.09(0.59)$ & $26.31(2.36)$ & $22.32(0.65)$ \\
\hline & 0.5 & $30.35(1.55)$ & $26.07(1.01)$ & $26.07(1.01)$ & $29.52(1.81)$ & $22.70(0.77)$ & $27.48(1.47)$ & $23.70(0.67)$ \\
\hline & 0.7 & $20.35(0.80)$ & $20.54(0.61)$ & $20.54(0.61)$ & $19.94(0.78)$ & $19.70(0.97)$ & $20.59(0.60)$ & $19.39(0.66)$ \\
\hline \multirow{3}{*}{ MNIST } & 0.3 & $24.58(2.82)$ & $17.99(1.44)$ & $17.99(1.44)$ & $22.18(2.75)$ & $20.92(0.74)$ & $12.74(0.63)$ & $11.76(0.78)$ \\
\hline & 0.5 & $23.00(1.60)$ & $22.35(1.10)$ & $22.35(1.10)$ & $23.55(1.80)$ & 42.10 (1.85) & $15.35(0.75)$ & 18.18 \\
\hline & 0.7 & $53.34(3.78)$ & $52.19(4.41)$ & $54.42(3.99)$ & $53.39(3.74)$ & $60.86(1.25)$ & $16.38(0.84)$ & $18.64(2.83)$ \\
\hline \multirow{3}{*}{ F-MNIST } & 0.3 & $14.88(1.30)$ & $18.02(2.86)$ & $18.02(2.86)$ & $15.12(1.18)$ & $19.24(0.91)$ & $14.54(1.14)$ & $13.54(0.75)$ \\
\hline & 0.5 & $13.40(0.69)$ & $12.05(0.96)$ & $12.05(0.96)$ & $13.22(0.62)$ & $37.73(1.56)$ & $12.15(0.48)$ & $14.10(1.16)$ \\
\hline & 0.7 & $9.94(1.30)$ & $8.89(0.84)$ & $8.89(0.84)$ & $8.54(0.84)$ & $55.65(2.14)$ & $8.65(0.83)$ & $9.29(0.47)$ \\
\hline \multirow{3}{*}{20 News } & 0.3 & $38.89(3.00)$ & $40.30(3.64)$ & $42.48(3.54)$ & $38.70(3.81)$ & $18.66(0.47)$ & $66.62(1.59)$ & $36.31(4.13)$ \\
\hline & 0.5 & $44.48(1.82)$ & $43.85(2.03)$ & $46.67(1.15)$ & $47.77(0.87)$ & $34.73(0.80)$ & $50.00(0.00)$ & $45.88(1.64)$ \\
\hline & 0.7 & $50.69(0.95)$ & $53.61(0.73)$ & $51.77(1.05)$ & $50.36(0.83)$ & $50.69(0.95)$ & $30.61(0.59)$ & $29.85(0.13)$ \\
\hline
\end{tabular}

Benchmark Data: Next we apply the PURL method to benchmark datasets. To obtain low-dimensional representation, we set $m=20$ and use a fullyconnected neural network with four layers $(d-60-20-1 ; \boldsymbol{v}$ is $d-60-20$ and $g$ is 20-1.) for $w$ except text classification dataset. For text classification datasets, we use another fully-connected neural network with four layers $(d-30-10-1)$ for $w$, i.e., $m=10$. ReLU is used as activation functions for hidden layers, and batch normalization (Ioffe and Szegedy, 2015) is applied to all hidden layers. Stochastic gradient descent is used for optimization with learning rate 0.001 . Also, weight decay with 0.0005 and gradient noise with 0.01 are applied. We iteratively update $w$ with four mini-batches and $\boldsymbol{v}$ with one mini-batch.

We compare the accuracy of class-prior estimation with and without dimension reduction. For comparison, we also consider PCA, FDA, and PNRL. For PCA, we vary the numbers of components as follows: $\lfloor d / 4\rfloor,\lfloor d / 2\rfloor$, and $\lfloor 3 d / 4\rfloor$, where $\lfloor\cdot\rfloor$ is the floor function. For FDA, the reduced dimension is 1 due to the property of FDA (Hastie et al., 2009) in which the reduced dimension becomes the minimum of $m$ or (the number of classes -1 ). The neural network for PNRL is the same as the one for the proposed method.

As a class-prior estimation method, we use the method based on the kernel mean embedding (KM) method proposed by Ramaswamy et al. (2016). With 
the estimated class-prior, we then train a fully-connected neural network with five layers ( $m$-300-300-300-1). ReLU is used as activation functions for hidden layers, and batch normalization is applied to all hidden layers. Except for text classification datasets, we train the neural networks by Adam (Kingma and Ba, 2015) until 200 epochs. For text classification datasets, we use AdaGrad (Duchi et al., 2011) and set the number of epochs to 300. For non-negative PU learning (Kiryo et al., 2017), we use the sigmoid loss function and set $\beta$ and $\gamma$ in the paper to 0 and 1 , respectively.

We use the ijcnn1, phishing, mushrooms, and a9a datasets taken from the LIBSVM webpage (Chang and Lin, 2011). Also, we use the MNIST (LeCun et al., 1998), Fashion-MNIST (F-MNIST) (Xiao et al., 2017), and 20 Newsgroups (Lang, 1995) datasets. For the MNIST and F-MNIST datasets, we divide the whole classes into 2 groups to make binary classification tasks. For the 20 Newsgroups dataset, we use the "com" topic as the positive class and the "sci" topic as the negative class, ${ }^{\S}$ and make 2000 -dimensional $t f$-idf vector. From the datasets, we draw $n_{\mathrm{P}}=1000$ positive and $n_{\mathrm{U}}=2000$ unlabeled samples. For validation, we use $n_{\mathrm{P}}=50$ and $n_{\mathrm{U}}=200$ samples.

Table 1 lists the average absolute error between the estimated class-prior and the true value. Overall, our proposed dimension reduction method tends to outperform other methods, meaning that our method provides useful lowdimensional representation. Except for the ijcnn1 dataset, the error of FDA tends to be larger than the other methods, implying that regarding $U$ data as $\mathrm{N}$ data does not help in class-prior estimation. For the mushrooms and a9a datasets, applying the unsupervised dimension reduction method, PCA, does not improve the estimation accuracy, while our method reduces the error of class-prior estimation. In particular, for the 20 Newsgroups dataset, the existing approaches (PCA, FDA, and PNRL) perform poorly. In contrast, applying our method significantly reduces the error of class-prior estimation.

Then, we summarize the average misclassification rates in Table 2. Since the accuracy of class-prior estimation is improved on the mushrooms and a9a datasets, the classification accuracy is also improved. In particular, the classification results on the 20 Newsgroups dataset with $\theta_{\mathrm{P}}=0.7$ are improved substantially. Overall, our proposed method tends to give the lower or comparable misclassification rates compared with the other methods.

\section{Conclusions}

In this paper, we proposed an information-theoretic representation learning method from positive and unlabeled (PU) data. Our method is based on the

\footnotetext{
${ }^{\S}$ See http://qwone.com/ jason/20Newsgroups/ for the details of topics.
} 
information maximization principle, and find low-dimensional representation maximally preserving a squared-loss variant of mutual information (SMI) between inputs and labels. Unlike the existing PU learning methods, since our representation learning method can be executed without knowing an estimate of the class-prior in advance, our method can also be used as preprocessing for the class-prior estimation method. Through numerical experiments, we demonstrated the effectiveness of our method.

\section{Acknowledgements}

TS was supported by KAKENHI 15J09111. GN was supported by the JST CREST JPMJCR1403. MS was supported by KAKENHI 17H01760. We thank Ikko Yamane, Ryuichi Kiryo, and Takeshi Teshima for their comments.

\section{References}

Abadi, M., Agarwal, A., Barham, P., Brevdo, E., Chen, Z., Citro, C., Corrado, G. S., Davis, A., Dean, J., Devin, M., Ghemawat, S., Goodfellow, I., Harp, A., Irving, G., Isard, M., Jia, Y., Jozefowicz, R., Kaiser, L., Kudlur, M., Levenberg, J., Mané, D., Monga, R., Moore, S., Murray, D., Olah, C., Schuster, M., Shlens, J., Steiner, B., Sutskever, I., Talwar, K., Tucker, P., Vanhoucke, V., Vasudevan, V., Viégas, F., Vinyals, O., Warden, P., Wattenberg, M., Wicke, M., Yu, Y., and Zheng, $\mathrm{X}$. TensorFlow: Large-scale machine learning on heterogeneous systems, 2015. URL https://www.tensorflow.org/. Software available from https://www.tensorflow.org/.

Basu, A., Harris, I. R., Hjort, N. L., and Jones, M. C. Robust and efficient estimation by minimising a density power divergence. Biometrika, 85(3): 549-559, 1998.

Bonnans, J. F. and Cominetti, R. Perturbed optimization in Banach spaces I: A general theory based on a weak directional constraint qualificationn; II: A theory based on a strong directional qualification condition; III: Semiinfinite optimization. SIAM Journal on Control and Optimization, 34(4):1151-1171, 1172-1189, and 1555-1567, 1996.

Bonnans, J. F. and Shapiro, A. Optimization problems with perturbations: A guided tour. SIAM Review, 40(2):228-264, 1998.

Boyd, S. and Vandenberghe, L. Convex Optimization. Cambridge University Press, New York, NY, USA, 2004. 
Chang, C.-C. and Lin, C.-J. LIBSVM: A library for support vector machines. ACM Transactions on Intelligent Systems and Technology, 2:1-27, 2011. Software available at http://www.csie.ntu.edu.tw/ cjlin/libsvm.

Chapelle, O., Schölkopf, B., and Zien, A., editors. Semi-Supervised Learning. MIT Press, 2006.

Cover, T. M. and Thomas, J. A. Elements of Information Theory. John Wiley \& Sons, Inc., Hoboken, NJ, USA, 2nd edition, 2006.

du Plessis, M. C. and Sugiyama, M. Class prior estimation from positive and unlabeled data. IEICE Transactions on Information and Systems, E97-D(5): 1358-1362, 2014.

du Plessis, M. C., Niu, G., and Sugiyama, M. Convex formulation for learning from positive and unlabeled data. In Proceedings of 32nd International Conference on Machine Learning, volume 37, pages 1386-1394, 2015.

du Plessis, M. C., Niu, G., and Sugiyama, M. Class-prior estimation for learning from positive and unlabeled data. Machine Learning, 106(4):463-492, 2017.

Duchi, J., Hazan, E., and Singer, Y. Adaptive subgradient methods for online learning and stochastic optimization. Journal of Machine Learning Research, 12(Jul):2121-2159, 2011.

Elkan, C. and Noto, K. Learning classifiers from only positive and unlabeled data. In Proceedings of the 14th ACM SIGKDD International Conference on Knowledge Discovery and Data Mining, pages 213-220, 2008.

Glorot, X., Bordes, A., and Bengio, Y. Deep sparse rectifier neural networks. In Proceedings of the Fourteenth International Conference on Artificial Intelligence and Statistics, pages 315-323, 2011.

Goodfellow, I., Bengio, Y., and Courville, A. Deep Learning. MIT Press, 2016.

Hastie, T., Tibshirani, R., and Friedman, J. The Elements of Statistical Learning. Springer-Verlag, New York, 2 edition, 2009.

Ioffe, S. and Szegedy, C. Batch normalization: Accelerating deep network training by reducing internal covariate shift. In Proceedings of the 32nd International Conference on Machine Learning, pages 448-456, 2015.

Jain, S., White, M., and Radivojac, P. Estimating the class prior and posterior from noisy positives and unlabeled data. In Advances in Neural Information Processing Systems 29, 2016. 
Jia, Y., Shelhamer, E., Donahue, J., Karayev, S., Long, J., Girshick, R., Guadarrama, S., and Darrell, T. Caffe: Convolutional architecture for fast feature embedding. In Proceedings of the 22nd ACM International Cconference on Multimedia, pages 675-678, 2014.

Kanamori, T., Hido, S., and Sugiyama, M. A least-squares approach to direct importance estimation. Journal of Machine Learning Research, 10:1391-1445, 2009.

Kanamori, T., Suzuki, T., and Sugiyama, M. Statistical analysis of kernel-based least-squares density-ratio estimation. Machine Learning, 86(3):335-367, 2012.

Keziou, A. Dual representation of $\varphi$-divergences and applications. Comptes Rendus Mathématique, 336(10):857-862, 2003.

Khan, S., Bandyopadhyay, S., Ganguly, A., and Saigal, S. Relative performance of mutual information estimation methods for quantifying the dependence among short and noisy data. Physical Review E, 76:026209, 2007.

Kingma, D. P. and Ba, J. Adam: A method for stochastic optimization. In Proceedings of 3rd International Conference on Learning Representations, 2015.

Kiryo, R., Niu, G., du Plessis, M. C., and Sugiyama, M. Positive-unlabeled learning with non-negative risk estimator. In Advances in Neural Information Processing Systems 30, pages 1674-1684, 2017.

Kraskov, A., Stögbauer, H., and Grassberger, P. Estimating mutual information. Physical Review E, 69(6):066138, 2004.

Krause, A., Perona, P., and Gomes, R. G. Discriminative clustering by regularized information maximization. In Advances in Neural Information Processing Systems, pages 775-783, 2010.

Lang, K. Newsweeder: Learning to filter netnews. In Proceedings of the Twelfth International Conference on Machine Learning, pages 331-339, 1995.

LeCun, Y., Bottou, L., Bengio, Y., and Haffner, P. Gradient-based learning applied to document recognition. Proceedings of the IEEE, 86(11):22782324, 1998.

Letouzey, F., Denis, F., and Gilleron, R. Learning from positive and unlabeled examples. In Proceedings of the 11th International Conference on Algorithmic 
Learning Theory, pages 71-85, Berlin, Heidelberg, 2000. Springer Berlin Heidelberg.

Li, K.-C. Sliced inverse regression for dimension reduction. Journal of the American Statistical Association, 86(414):316-327, 1991.

Li, W., Guo, Q., and Elkan, C. A positive and unlabeled learning algorithm for one-class classification of remote-sensing data. IEEE Transactions on Geoscience and Remote Sensing, 49(2):717-725, 2011.

Linsker, R. Self-organization in a perceptual network. Computer, 21(3):105-117, 1988.

Mohri, M., Rostamizadeh, A., and Talwalkar, A. Foundations of Machine Learning. MIT Press, 2012.

Moon, Y.-I., Rajagopalan, B., and Lall, U. Estimation of mutual information using kernel density estimators. Physical Review E, 52(3):2318-2321, 1995.

Nguyen, X. L., Wainwright, M. J., and Jordan, M. I. Nonparametric estimation of the likelihood ratio and divergence functionals. In IEEE International Symposium on Information Theory, pages 2016-2020, 2007.

Northcutt, C. G., Wu, T., and Chuang, I. L. Learning with confident examples: Rank pruning for robust classification with noisy labels. In Proceedings of the Thirty-Third Conference on Uncertainty in Artificial Intelligence, 2017.

Pearson, K. On the criterion that a given system of deviations from the probable in the case of a correlated system of variables is such that it can be reasonably supposed to have arise from random sampling. Philosophical Magazine Series 5, 50(302):157-175, 1990.

Ramaswamy, H. G., Scott, C., and Tewari, A. Mixture proportion estimation via kernel embedding of distributions. In Proceedings of the 33rd International Conference on Machine Learning, 2016.

Sakai, T. and Sugiyama, M. Computationally efficient estimation of squared-loss mutual information with multiplicative kernel models. IEICE Transactions on Information and Systems, E97-D(4):968-971, 2014.

Sugiyama, M. Machine learning with squared-loss mutual information. Entropy, 15:80-112, 2013. 
Sugiyama, M., Suzuki, T., and Kanamori, T. Density ratio matching under the Bregman divergence: A unified framework of density ratio estimation. Annals of the Institute of Statistical Mathematics, 64(5):1009-1044, 2012a.

Sugiyama, M., Suzuki, T., and Kanamori, T. Density Ratio Estimation in Machine Learning. Cambridge University Press, Cambridge, UK, 2012b.

Suzuki, T. and Sugiyama, M. Sufficient dimension reduction via squared-loss mutual information. Neural Computation, 25(3):725-758, 2013.

Suzuki, T., Sugiyama, M., Sese, J., and Kanamori, T. Approximating mutual information by maximum likelihood density ratio estimation. In Proceedings of ECML-PKDD2008 Workshop on New Challenges for Feature Selection in Data Mining and Knowledge Discovery (FSDM2008), volume 4, pages 5-20, Antwerp, Belgium, Sep. 152008.

Suzuki, T., Sugiyama, M., Kanamori, T., and Sese, J. Mutual information estimation reveals global associations between stimuli and biological processes. BMC Bioinformatics, 10:S52:1-12, 2009.

Torkkola, K. Feature extraction by non-parametric mutual information maximization. Journal of Machine Learning Research, 3:1415-1438, 2003.

Van Hulle, M. M. Edgeworth approximation of multivariate differential entropy. Neural Computation, 17(9):1903-1910, 2005.

Ward, G., Hastie, T., Barry, S., Elith, J., and Leathwick, J. R. Presence-only data and the EM algorithm. Biometrics, 65(2):554-563, 2009.

Xiao, H., Rasul, K., and Vollgraf, R. Fashion-MNIST: a novel image dataset for benchmarking machine learning algorithms. arXiv, 2017. 


\section{A Proof of Theorem 1}

Proof. Let us express SMI in Eq. (1) as

$\mathrm{SMI}=\frac{\theta_{\mathrm{P}}}{2} \int\left(\frac{p(\boldsymbol{x} \mid y=+1)}{p(\boldsymbol{x})}-1\right)^{2} p(\boldsymbol{x}) \mathrm{d} \boldsymbol{x}+\frac{\theta_{\mathrm{N}}}{2} \int\left(\frac{p(\boldsymbol{x} \mid y=-1)}{p(\boldsymbol{x})}-1\right)^{2} p(\boldsymbol{x}) \mathrm{d} \boldsymbol{x}$.

From the marginal density $p(\boldsymbol{x})$, we have

$$
\begin{aligned}
\theta_{\mathrm{N}} \frac{p(\boldsymbol{x} \mid y=-1)}{p(\boldsymbol{x})} & =1-\theta_{\mathrm{P}} \frac{p(\boldsymbol{x} \mid y=+1)}{p(\boldsymbol{x})}, \\
\theta_{\mathrm{N}}\left(\frac{p(\boldsymbol{x} \mid y=-1)}{p(\boldsymbol{x})}-1\right) & =\theta_{\mathrm{P}}\left(1-\frac{p(\boldsymbol{x} \mid y=+1)}{p(\boldsymbol{x})}\right), \\
\left(\frac{p(\boldsymbol{x} \mid y=-1)}{p(\boldsymbol{x})}-1\right)^{2} & =\frac{\theta_{\mathrm{P}}^{2}}{\theta_{\mathrm{N}}^{2}}\left(\frac{p(\boldsymbol{x} \mid y=+1)}{p(\boldsymbol{x})}-1\right)^{2},
\end{aligned}
$$

where the equality between the first and second equations can be confirmed by using $\theta_{\mathrm{P}}+\theta_{\mathrm{N}}=1$. Plugging the last equation into the second term of Eq. (8), we then obtain an expression of SMI only with positive and unlabeled data (PU-SMI) as

$$
\mathrm{SMI}=\frac{\theta_{\mathrm{P}}}{2 \theta_{\mathrm{N}}} \int\left(\frac{p(\boldsymbol{x} \mid y=+1)}{p(\boldsymbol{x})}-1\right)^{2} p(\boldsymbol{x}) \mathrm{d} \boldsymbol{x}=: \text { PU-SMI. }
$$

\section{B Proof of Theorem 2}

Proof. Let

$$
s(\boldsymbol{x}):=\frac{p(\boldsymbol{x} \mid y=+1)}{p(\boldsymbol{x})}
$$

be the density ratio. Then, PU-SMI can be expressed as

$$
\begin{aligned}
\text { PU-SMI } & =\frac{\theta_{\mathrm{P}}}{2 \theta_{\mathrm{N}}} \int(s(\boldsymbol{x})-1)^{2} p(\boldsymbol{x}) \mathrm{d} \boldsymbol{x} \\
& =\frac{\theta_{\mathrm{P}}}{\theta_{\mathrm{N}}}\left(\frac{1}{2} \int s^{2}(\boldsymbol{x}) p(\boldsymbol{x}) \mathrm{d} \boldsymbol{x}-\frac{1}{2}\right),
\end{aligned}
$$


where $s(\boldsymbol{x}) p(\boldsymbol{x})=p(\boldsymbol{x} \mid y=+1)$ is used. Based on the Fenchel inequality (Boyd and Vandenberghe, 2004), for any function $f(\boldsymbol{x})$ in a function class $\mathcal{F}$, we have

$$
\frac{1}{2} s^{2}(\boldsymbol{x}) \geq f(\boldsymbol{x}) s(\boldsymbol{x})-\frac{1}{2} f^{2}(\boldsymbol{x}) .
$$

Then, we obtain the lower bound of the PU-SMI by

$$
\begin{aligned}
\text { PU-SMI } & \geq \frac{\theta_{\mathrm{P}}}{\theta_{\mathrm{N}}}\left(\int f(\boldsymbol{x}) p(\boldsymbol{x} \mid y=+1) \mathrm{d} \boldsymbol{x}-\frac{1}{2} \int f^{2}(\boldsymbol{x}) p(\boldsymbol{x}) \mathrm{d} \boldsymbol{x}-\frac{1}{2}\right) \\
& =\frac{\theta_{\mathrm{P}}}{\theta_{\mathrm{N}}}\left(-J_{\mathrm{PU}}(f)-\frac{1}{2}\right),
\end{aligned}
$$

where

$$
J_{\mathrm{PU}}(f):=\frac{1}{2} \int f^{2}(\boldsymbol{x}) p(\boldsymbol{x}) \mathrm{d} \boldsymbol{x}-\int f(\boldsymbol{x}) p(\boldsymbol{x} \mid y=+1) \mathrm{d} \boldsymbol{x} .
$$

Thus, from the Fenchel duality (Keziou, 2003; Nguyen et al., 2007), we have

$$
\mathrm{PU}-\mathrm{SMI}=\sup _{f \in \mathcal{F}} \frac{\theta_{\mathrm{P}}}{\theta_{\mathrm{N}}}\left(-J_{\mathrm{PU}}(f)-\frac{1}{2}\right),
$$

where equality in the supremum is attained when $f(\boldsymbol{x})=s(\boldsymbol{x})=p(\boldsymbol{x} \mid y=$ $+1) / p(\boldsymbol{x})$ and $s \in \mathcal{F}$.

\section{Proof of Theorem 3}

The idea of the proof is to view the approximated squared error as perturbed optimization of expected one. In the analysis, we focus on the linear-in-parameter model $w(\boldsymbol{x})=\sum_{\ell=1}^{b} \beta_{\ell} \phi_{\ell}(\boldsymbol{x})=\boldsymbol{\beta}^{\top} \boldsymbol{\phi}(\boldsymbol{x})$. We assume that $0 \leq \phi_{\ell} \leq 1$ for all $\ell=1, \ldots, b$ and $\boldsymbol{x} \in \mathbb{R}^{d}$, and $\widehat{\boldsymbol{H}}^{\mathrm{U}}$ and $\boldsymbol{H}^{\mathrm{U}}$ are positive definite matrices. Recall

$$
\boldsymbol{\beta}^{*}=\underset{\boldsymbol{\beta} \in \mathbb{R}^{b}}{\operatorname{argmin}} J_{\mathrm{PU}}(\boldsymbol{\beta}),
$$

where

$$
\begin{aligned}
J_{\mathrm{PU}}(\boldsymbol{\beta}) & =\frac{1}{2} \boldsymbol{\beta}^{\top} \boldsymbol{H}^{\mathrm{U}} \boldsymbol{\beta}^{\top}-\boldsymbol{\beta}^{\top} \boldsymbol{h}^{\mathrm{P}}, \\
\boldsymbol{H}^{\mathrm{U}} & =\int \boldsymbol{\phi}(\boldsymbol{x}) \boldsymbol{\phi}(\boldsymbol{x})^{\top} p(\boldsymbol{x}) \mathrm{d} \boldsymbol{x}, \\
\boldsymbol{h}^{\mathrm{P}} & =\int \boldsymbol{\phi}(\boldsymbol{x}) p(\boldsymbol{x} \mid y=+1) \mathrm{d} \boldsymbol{x} .
\end{aligned}
$$


Similarly,

$$
\widehat{\boldsymbol{\beta}}=\underset{\boldsymbol{\beta} \in \mathbb{R}^{b}}{\operatorname{argmin}} \widehat{J}_{\mathrm{PU}}(\boldsymbol{\beta})
$$

where

$$
\begin{aligned}
\widehat{J}_{\mathrm{PU}}(\boldsymbol{\beta}) & =\frac{1}{2} \boldsymbol{\beta}^{\top} \widehat{\boldsymbol{H}}^{\mathrm{U}} \boldsymbol{\beta}^{\top}-\boldsymbol{\beta}^{\top} \widehat{\boldsymbol{h}}^{\mathrm{P}}, \\
\widehat{\boldsymbol{H}}^{\mathrm{U}} & =\frac{1}{n_{\mathrm{U}}} \sum_{k=1}^{n_{\mathrm{U}}} \boldsymbol{\phi}\left(\boldsymbol{x}_{k}^{\mathrm{U}}\right) \boldsymbol{\phi}\left(\boldsymbol{x}_{k}^{\mathrm{U}}\right)^{\top}, \\
\widehat{\boldsymbol{h}}^{\mathrm{P}} & =\frac{1}{n_{\mathrm{P}}} \sum_{i=1}^{n_{\mathrm{P}}} \boldsymbol{\phi}\left(\boldsymbol{x}_{i}^{\mathrm{P}}\right) .
\end{aligned}
$$

Firstly, we have the following lemma:

Lemma 4. Let $\epsilon$ be the smallest eigenvalue of $\boldsymbol{H}^{\mathrm{U}}$. We have

$$
J_{\mathrm{PU}}(\boldsymbol{\beta}) \geq J_{\mathrm{PU}}\left(\boldsymbol{\beta}^{*}\right)+\epsilon\left\|\boldsymbol{\beta}-\boldsymbol{\beta}^{*}\right\|_{2}^{2} .
$$

That is, $J_{\mathrm{PU}}$ satisfies the second order growth condition (Bonnans and Shapiro, 1998).

Proof. Since $\boldsymbol{H}^{\mathrm{U}}$ is positive definite, $J_{\mathrm{PU}}(\boldsymbol{\beta})$ is strongly convex with parameter at least $\epsilon$. Then, we have

$$
\begin{aligned}
J_{\mathrm{PU}}(\boldsymbol{\beta}) & \geq J_{\mathrm{PU}}\left(\boldsymbol{\beta}^{*}\right)+\nabla J_{\mathrm{PU}}\left(\boldsymbol{\beta}^{*}\right)^{\top}\left(\boldsymbol{\beta}-\boldsymbol{\beta}^{*}\right)+\epsilon\left\|\boldsymbol{\beta}-\boldsymbol{\beta}^{*}\right\|_{2}^{2} \\
& =J_{\mathrm{PU}}\left(\boldsymbol{\beta}^{*}\right)+\epsilon\left\|\boldsymbol{\beta}-\boldsymbol{\beta}^{*}\right\|_{2}^{2},
\end{aligned}
$$

where the optimality condition $\nabla J_{\mathrm{PU}}\left(\boldsymbol{\beta}^{*}\right)=\mathbf{0}$ is used.

Let us define a set of perturbation parameters as

$$
\mathcal{U}:=\left\{\boldsymbol{U}^{\mathrm{U}}, \boldsymbol{u}^{\mathrm{P}} \mid \boldsymbol{U}^{\mathrm{U}} \in \mathbb{S}^{b}, \boldsymbol{u}^{\mathrm{P}} \in \mathbb{R}^{b}\right\},
$$

where $\mathbb{S}^{b}$ is the set of symetric $b \times b$ matrices. With these perturbation parameters, we express $\widehat{\boldsymbol{H}}^{\mathrm{U}}$ and $\widehat{\boldsymbol{h}}^{\mathrm{P}}$ as $\boldsymbol{U}^{\mathrm{U}}=\widehat{\boldsymbol{H}}^{\mathrm{U}}-\boldsymbol{H}^{\mathrm{U}}$ and $\boldsymbol{u}^{\mathrm{P}}=\widehat{\boldsymbol{h}}^{\mathrm{P}}-\boldsymbol{h}^{\mathrm{P}}$, respectively. Let $\boldsymbol{u} \in \mathcal{U}$. Our perturbed objective function and the solution are given by

$$
\begin{aligned}
J_{\mathrm{PU}}(\boldsymbol{\beta}, \boldsymbol{u}) & :=\frac{1}{2} \boldsymbol{\beta}^{\top}\left(\boldsymbol{H}^{\mathrm{U}}+\boldsymbol{U}^{\mathrm{U}}\right) \boldsymbol{\beta}-\boldsymbol{\beta}^{\top}\left(\boldsymbol{h}^{\mathrm{P}}+\boldsymbol{u}^{\mathrm{P}}\right), \\
\boldsymbol{\beta}(\boldsymbol{u}) & :=\underset{\boldsymbol{\beta} \in \mathbb{R}^{b}}{\operatorname{argmin}} J_{\mathrm{PU}}(\boldsymbol{\beta}, \boldsymbol{u}) .
\end{aligned}
$$

Apparently, $J_{\mathrm{PU}}(\boldsymbol{\beta})=J_{\mathrm{PU}}(\boldsymbol{\beta}, \mathbf{0})$. Also, $\widehat{J}_{\mathrm{PU}}(\boldsymbol{\beta})=J_{\mathrm{PU}}(\boldsymbol{\beta}, \boldsymbol{u})$ and $\widehat{\boldsymbol{\beta}}=\boldsymbol{\beta}(\boldsymbol{u})$ for $\boldsymbol{u} \neq \mathbf{0}$. We then have the following Lemma: 
Lemma 5. $J_{\mathrm{PU}}(\cdot, \boldsymbol{u})-J_{\mathrm{PU}}(\cdot)$ is Lipschitz continuous modulus $\omega(\boldsymbol{u})=$ $\mathcal{O}\left(\left\|\boldsymbol{U}^{\mathrm{U}}\right\|_{\text {Fro }}+\left\|\boldsymbol{u}^{\mathrm{P}}\right\|_{2}\right)$, where $\|\cdot\|_{\text {Fro }}$ is the Frobenius norm.

Proof. Firstly, we have

$$
J_{\mathrm{PU}}(\boldsymbol{\beta}, \boldsymbol{u})-J_{\mathrm{PU}}(\boldsymbol{\beta})=\frac{1}{2} \boldsymbol{\beta}^{\top} \boldsymbol{U}^{\mathrm{U}} \boldsymbol{\beta}-\boldsymbol{\beta}^{\top} \boldsymbol{u}^{\mathrm{P}} .
$$

The partial gradient is given by

$$
\frac{\partial}{\partial \boldsymbol{\beta}}\left(J_{\mathrm{PU}}(\boldsymbol{\beta}, \boldsymbol{u})-J_{\mathrm{PU}}(\boldsymbol{\beta})\right)=\boldsymbol{U}^{\mathrm{U}} \boldsymbol{\beta}-\boldsymbol{u}^{\mathrm{P}} .
$$

Let us define the $\delta$-ball of $\boldsymbol{\beta}^{*}$ as $\mathcal{B}_{\delta}\left(\boldsymbol{\beta}^{*}\right):=\left\{\boldsymbol{\beta} \mid\left\|\boldsymbol{\beta}-\boldsymbol{\beta}^{*}\right\|_{2} \leq \delta\right\}$, and $M=$ $\left\|\boldsymbol{\beta}^{*}\right\|_{2}$. For any $\boldsymbol{\beta} \in \mathcal{B}_{\delta}\left(\boldsymbol{\beta}^{*}\right)$, we can easily show

$$
\|\boldsymbol{\beta}\|_{2} \leq\left\|\boldsymbol{\beta}-\boldsymbol{\beta}^{*}\right\|_{2}+\left\|\boldsymbol{\beta}^{*}\right\|_{2} \leq \delta+M,
$$

where we first used the triangle inequality and then $\left\|\boldsymbol{\beta}-\boldsymbol{\beta}^{*}\right\|_{2} \leq \delta$ and $M=$ $\left\|\boldsymbol{\beta}^{*}\right\|_{2}$. Thus,

$$
\left\|\frac{\partial}{\partial \boldsymbol{\beta}}\left(J_{\mathrm{PU}}(\boldsymbol{\beta}, \boldsymbol{u})-J_{\mathrm{PU}}(\boldsymbol{\beta})\right)\right\|_{2} \leq(\delta+M)\left\|\boldsymbol{U}^{\mathrm{U}}\right\|_{\text {Fro }}+\left\|\boldsymbol{u}^{\mathrm{P}}\right\|_{2} .
$$

This means that $J_{\mathrm{PU}}(\cdot, \boldsymbol{u})-J_{\mathrm{PU}}(\cdot)$ is Lipschitz continuous on $\mathcal{B}_{\delta}\left(\boldsymbol{\beta}^{*}\right)$ with a Lipschitz constant of order $\mathcal{O}\left(\left\|\boldsymbol{U}^{\mathrm{U}}\right\|_{\text {Fro }}+\left\|\boldsymbol{u}^{\mathrm{P}}\right\|_{2}\right)$.

Finally, we prove Theorem 3.

Proof. According to the central limit theorem, we have

$$
\left\|\boldsymbol{U}^{\mathrm{U}}\right\|_{\text {Fro }}=\mathcal{O}_{p}\left(1 / \sqrt{n_{\mathrm{U}}}\right), \quad\left\|\boldsymbol{u}^{\mathrm{P}}\right\|_{2}=\mathcal{O}_{p}\left(1 / \sqrt{n_{\mathrm{P}}}\right)
$$

as $n_{\mathrm{P}}, n_{\mathrm{U}} \rightarrow \infty$. Since we proved that $J_{\mathrm{PU}}$ satisfies the second order growth condition (Lemma 4) and $J_{\mathrm{PU}}(\cdot, \boldsymbol{u})-J_{\mathrm{PU}}(\cdot)$ is Lipschitz continuous modulus $\omega(\boldsymbol{u})$ (Lemma 5), we can use Proposition 6.1 in Bonnans and Shapiro (1998) and have the first half of Theorem 3:

$$
\begin{aligned}
\left\|\widehat{\boldsymbol{\beta}}-\boldsymbol{\beta}^{*}\right\|_{2} & \leq \epsilon^{-1} \omega(\boldsymbol{u}) \\
& =\mathcal{O}\left(\left\|\boldsymbol{U}^{\mathrm{U}}\right\|_{\text {Fro }}+\left\|\boldsymbol{u}^{\mathrm{P}}\right\|_{2}\right) \\
& =\mathcal{O}_{p}\left(1 / \sqrt{n_{\mathrm{P}}}+1 / \sqrt{n_{\mathrm{U}}}\right) .
\end{aligned}
$$

Next, we prove the latter half of Theorem 3. For the squared errors, we have

$$
\left|\widehat{J}_{\mathrm{PU}}(\widehat{\boldsymbol{\beta}})-J_{\mathrm{PU}}\left(\boldsymbol{\beta}^{*}\right)\right| \leq\left|\widehat{J}_{\mathrm{PU}}(\widehat{\boldsymbol{\beta}})-\widehat{J}_{\mathrm{PU}}\left(\boldsymbol{\beta}^{*}\right)\right|+\left|\widehat{J}_{\mathrm{PU}}\left(\boldsymbol{\beta}^{*}\right)-J_{\mathrm{PU}}\left(\boldsymbol{\beta}^{*}\right)\right| \text {. }
$$


Here, we have

$$
\begin{aligned}
\widehat{J}_{\mathrm{PU}}(\widehat{\boldsymbol{\beta}})-\widehat{J}_{\mathrm{PU}}\left(\boldsymbol{\beta}^{*}\right) & =\frac{1}{2}\left(\widehat{\boldsymbol{\beta}}+\boldsymbol{\beta}^{*}\right)^{\top} \widehat{\boldsymbol{H}}^{\mathrm{U}}\left(\widehat{\boldsymbol{\beta}}-\boldsymbol{\beta}^{*}\right)-\left(\widehat{\boldsymbol{\beta}}-\boldsymbol{\beta}^{*}\right)^{\top} \widehat{\boldsymbol{h}}^{\mathrm{P}}, \\
\widehat{J}_{\mathrm{PU}}\left(\boldsymbol{\beta}^{*}\right)-J_{\mathrm{PU}}\left(\boldsymbol{\beta}^{*}\right) & =\frac{1}{2} \boldsymbol{\beta}^{* \top} \boldsymbol{U}^{\mathrm{U}} \boldsymbol{\beta}^{*}-\boldsymbol{u}^{\mathrm{P}} \boldsymbol{\beta}^{*}
\end{aligned}
$$

Since $0 \leq \phi_{\ell}(\boldsymbol{x}) \leq 1$ and $M=\left\|\boldsymbol{\beta}^{*}\right\|_{2}$, it leads to

$$
\begin{aligned}
\left|\widehat{J}_{\mathrm{PU}}(\widehat{\boldsymbol{\beta}})-J_{\mathrm{PU}}\left(\boldsymbol{\beta}^{*}\right)\right| & \leq\left|\widehat{J}_{\mathrm{PU}}(\widehat{\boldsymbol{\beta}})-\widehat{J}_{\mathrm{PU}}\left(\boldsymbol{\beta}^{*}\right)\right|+\left|\widehat{J}_{\mathrm{PU}}\left(\boldsymbol{\beta}^{*}\right)-J_{\mathrm{PU}}\left(\boldsymbol{\beta}^{*}\right)\right| \\
& \leq \mathcal{O}_{p}\left(\left\|\widehat{\boldsymbol{\beta}}-\boldsymbol{\beta}^{*}\right\|_{2}\right)+\mathcal{O}_{p}\left(\left\|\boldsymbol{U}^{\mathrm{U}}\right\|_{\mathrm{Fro}}+\left\|\boldsymbol{u}^{\mathrm{P}}\right\|_{2}\right) \\
& =\mathcal{O}_{p}\left(1 / \sqrt{n_{\mathrm{P}}}+1 / \sqrt{n_{\mathrm{U}}}\right) .
\end{aligned}
$$

Recall

$$
\begin{aligned}
\mathrm{PU}_{-S M I}^{*} & =\frac{\theta_{\mathrm{P}}}{\theta_{\mathrm{N}}}\left(-J_{\mathrm{PU}}\left(\boldsymbol{\beta}^{*}\right)-\frac{1}{2}\right), \\
\widehat{\mathrm{PU}-\mathrm{SMI}} & =\frac{\theta_{\mathrm{P}}}{\theta_{\mathrm{N}}}\left(-\widehat{J}_{\mathrm{PU}}(\widehat{\boldsymbol{\beta}})-\frac{1}{2}\right) .
\end{aligned}
$$

We thus have

$$
\begin{aligned}
\left|\mathrm{PU}_{-S M I}{ }^{*}-\widehat{\mathrm{PU}-\mathrm{SM}}\right| & =\frac{\theta_{\mathrm{P}}}{\theta_{\mathrm{N}}}\left|\widehat{J}_{\mathrm{PU}}(\widehat{\boldsymbol{\beta}})-J_{\mathrm{PU}}\left(\boldsymbol{\beta}^{*}\right)\right| \\
& =\mathcal{O}_{p}\left(1 / \sqrt{n_{\mathrm{P}}}+1 / \sqrt{n_{\mathrm{U}}}\right) .
\end{aligned}
$$

This concludes the theorem.

\section{Effect of Dimension Reduction}

In this section, we illustrate the effect of dimension reduction and how the number of samples affects class-prior estimation.

We use the artificial dataset used in Section 5.2 and vary both $n_{\mathrm{P}}$ and $n_{\mathrm{U}}$ from 500 to 5,000 . We set the true class-prior $\theta_{\mathrm{P}}$ as 0.5 . The class-prior is estimated by the method based on kernel mean embedding (KM) (Ramaswamy et al., 2016). To evaluate the performance with and without dimension reduction, we use the one-dimensional samples obtained by $\boldsymbol{b}^{\top} \boldsymbol{x}$, where $\boldsymbol{b}=(1,0)^{\top}$, and the original two-dimensional samples.

Figure 3(a) shows the mean absolute error (with its standard error) between the true and estimated class-priors over 10 trials. The error of KM without dimension reduction decreases the number of samples until around $n_{\mathrm{P}}=n_{\mathrm{U}}=$ 2,000 , but the error is not reduced even if we increase $n_{\mathrm{P}}=n_{\mathrm{U}}=5,000$. In 


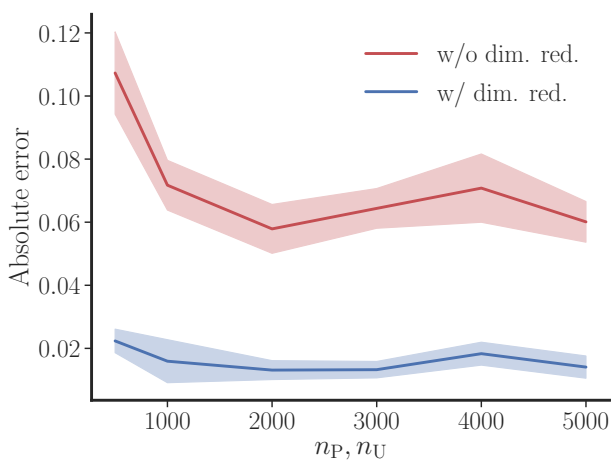

(a) Mean absolute error (with its standard error) between the true and estimated class-priors over 10 trials.

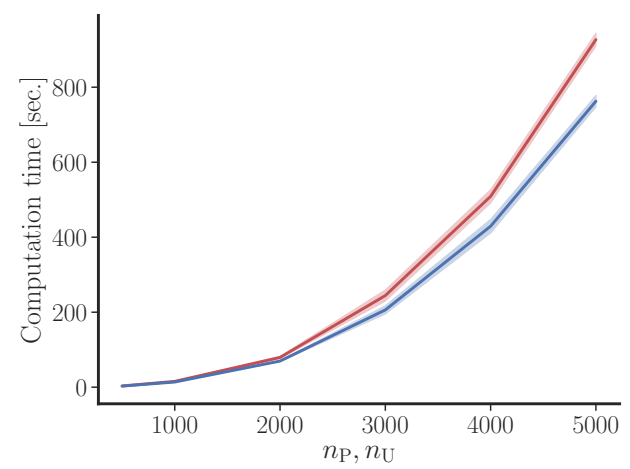

(b) Mean computation time [sec.] (with its standard error) over 10 trials.

Figure 3: Although the error of $\mathrm{KM}$ without dimension reduction decreases the number of samples until around $n_{\mathrm{P}}=n_{\mathrm{U}}=2,000$, the error of $\mathrm{KM}$ with dimension reduction is smaller than that without dimension reduction. For the computation time, it grows with the number of samples. Since the short computation time and low absolute error are desirable, this result shows the effectiveness of dimension reduction.

contrast, at $n_{\mathrm{P}}=n_{\mathrm{U}}=500$, the error of $\mathrm{KM}$ with dimension reduction is already smaller than that without dimension reduction. Figure 3(b) shows the mean computation time (with its standard error) over 10 trials. The computation time grows with the number of samples. Since the short computation time and low absolute error are desirable, this result shows the effectiveness of dimension reduction.

\section{E Supervised counterpart of the proposed method}

In this section, we review the SMI estimation method (Suzuki et al., 2009; Sugiyama, 2013; Sakai and Sugiyama, 2014).

According to Suzuki and Sugiyama (2013), SMI can be exrepssed as

$$
\mathrm{SMI}=\frac{1}{2} \sum_{y\{ \pm 1\}} \int r^{2}(\boldsymbol{x}, y) p(\boldsymbol{x}) p(y) \mathrm{d} \boldsymbol{x}-\frac{1}{2},
$$

where

$$
r(\boldsymbol{x}, y):=\frac{p(\boldsymbol{x}, y)}{p(\boldsymbol{x}) p(y)} .
$$


Based on the Fnechel inequality (Boyd and Vandenberghe, 2004), for any function $h: \mathbb{R}^{d} \times\{ \pm 1\} \rightarrow \mathbb{R}$, we have

$$
\frac{1}{2} r^{2}(\boldsymbol{x}, y) \geq h(\boldsymbol{x}, y) r(\boldsymbol{x}, y)-\frac{1}{2} h^{2}(\boldsymbol{x}, y),
$$

where the equality condition is $h(\boldsymbol{x}, y)=r(\boldsymbol{x}, y)$. We thus obtain the lower bound of SMI by

$$
\mathrm{SMI} \geq L(h)-\frac{1}{2}
$$

where

$$
L(h):=\sum_{y \in\{ \pm 1\}} \int h(\boldsymbol{x}, y) p(\boldsymbol{x}, y) \mathrm{d} \boldsymbol{x}-\frac{1}{2} \sum_{y \in\{ \pm 1\}} \int h^{2}(\boldsymbol{x}, y) p(\boldsymbol{x}) p(y) \mathrm{d} \boldsymbol{x} .
$$

To obtain an SMI estimate, we first train $h$ with PN data by solving the following optimization problem:

$$
\underset{h \in \mathcal{H}}{\operatorname{maximize}} \widehat{L}(h)
$$

where $\mathcal{H}$ is a user-specified function class and $\widehat{L}$ is sample approximation of $L$, i.e.,

$$
\widehat{L}(h):=\frac{1}{n} \sum_{i=1}^{n} h\left(\boldsymbol{x}_{i}, y_{i}\right)-\sum_{y \in\{ \pm 1\}} \frac{\widehat{p}(y)}{2 n} \sum_{i=1}^{n} h^{2}\left(\boldsymbol{x}_{i}, y\right)-\frac{1}{2} .
$$

A simple approach to approxiamtion of $\widehat{p}(y)$ is to use the number of labeled samples, i.e., $\widehat{p}(y=+1)$ is approximated by the number of positive samples divided by that of all labeled samples. The SMI approximator from PN data is then given by

$$
\widehat{\mathrm{PN}-\mathrm{SMI}}:=\widehat{L}(\widehat{h})-\frac{1}{2}
$$

where $\widehat{h}:=\operatorname{argmax}_{h \in \mathcal{H}} \widehat{L}(h)$.

Similarly to the proposed PURL method, we maximize PN-SMI to learn low-dimensional representation. 